\title{
Association of energy adjusts Nutrient-rich foods (ENRF) on mental health among obese and overweight women: a cross-sectional study
}

\section{Zahra Salehi}

Tehran University of Medical Sciences

\section{Farideh Shiraseb}

Tehran University of Medical Sciences

\section{Niloufar Rasaei}

Tehran University of Medical Sciences

Khadijeh Mirzaei ( $\boldsymbol{\theta}$ mirzaei_kh@tums.ac.ir)

School of Nutritional Sciences and Dietetics, Tehran University of Medical Science, Tehran, Iran https://orcid.org/0000-0003-0231-0478

\section{Research}

Keywords: ENRF9.3 index score, Mental health, Overweight, Obesity, Stress, Anxiety, Depression

Posted Date: May 11th, 2021

DOI: https://doi.org/10.21203/rs.3.rs-488142/v1

License: (a) (1) This work is licensed under a Creative Commons Attribution 4.0 International License. Read Full License 


\section{Abstract \\ Background}

Mental health and obesity have a bilateral relationship with each other. No study has been done on the relation between mental health and the ENRF9.3 index so far. Therefore, for the first time, we examined the relationship between the ENRF9.3 index and mental health in women with overweight and obesity.

\section{Methods}

In this cross-sectional study, 124 overweight and obese women were selected. Food intakes in the last 1 year were collected with 147 items of semi-quantitative food frequency. Then, the ENRF9.3 index score was calculated for all individuals. Mental health was assessed with a 21-item Depression Anxiety Stress Scales (DASS) questionnaire.

\section{Results}

In the present study, before adjusting for potential confounders the total DASS score was marginal significance $(P=0.05)$, that after the adjustment, it became insignificant $(P>0.05)$, after adjusting for confounders stress also became significant $(P=0.04)$. But no significant relationship was observed between depression and anxiety, even after adjustment $(P>0.05)$.

\section{Conclusions}

The present study showed that a nutritious diet is not associated with overall mental health score, among subgroups of the DASS total score, the relationship was seen only for the stress subgroup.

\section{Background}

Obesity is increasing rapidly worldwide, and this difficulty has doubled since 1980 . The World Health Organization estimates that by 2025 , one in five adults will be obese [1, 2]. In Iran, the prevalence of a combination of obesity and overweight may be more than $76 \%$ in some areas[2] Obesity is more common in women(39.5\%) than men(14.5\%)[3]. The interactive effect of positive energy balance, metabolic, genetic, environmental, and psychological factors may play a decisive role in the etiology of obesity[4, 7]. Progressive weight gain can cause a range of comorbidities such as dyslipidemia, hypertension, cardiovascular disease, and certain types of cancer[8]. The risk of mental illness in individuals with obesity has been documented to range between $30-70 \%[9]$ Some evidence has indicated that the relationship between weight and mental illness is dose-dependent, higher BMI increases the susceptibility for an incident psychiatric disorder and reverses [10, 11].

The common psychological disorders including depression and anxiety have a considerable contribution to the global burden of disease, accounting for $7.4 \%$ of all healthy years of life lost[12]. Among the Iranian population, $21 \%$ of adults are affected by mental disorders[13]. The complex interactions of social, environmental, and biological factors are contributing to psychological disorders[14]. Diet has been considered as a modifiable 
factor that plays a key role [15]. Including healthy diets such as the Japanese diet or Mediterranean diet, are inversely associated with depression risk [16]. While a pro-inflammatory diet increases the risk of adverse mental health consequences[17] such as high-fat meals could intensify stress[18].

Diet quality, which encompasses adequacy or sufficiency, moderation, diversity, and balance or equilibrium of nutrient and food intakes, is also critical to mental health [12]. One of the Nutrient quality models is the nutrientrich food(NRF) index[19]. The NRF index, unlike the quality of the general diet index, similar to the healthy eating index(HEI), has been tested and validated[20,21]. The NRF 9.3 index is based on 9 nutrients that are encouraged, and 3 nutrients that are restricted [22]. The association between the NRF index, all-cause mortality, incident cardiovascular diseases[23], various anthropometric measures [24], and malnutrition in children[19] have been assessed. No study has been performed on the Association of Nutrient-rich foods on mental health among obese and overweight women, therefore a cross-sectional study has been designed to determine this relationship.

\section{Methods And Materials}

\section{Study population}

A cross-sectional study was conducted on 124 health obese and overweight women who were referred to health centers in Tehran, Iran recruited by the multi-stage cluster random sampling method. Finally, 20 clusters were selected, because different socio-economic statuses were also considered. Before the study, all participants signed informed written consent. The sample entry criteria were met, including the range of BMI $25-34.9 \mathrm{~kg} / \mathrm{m}^{2}$ and the age between 18 - 48 years old. Also excluded people who met these criteria: pregnancy, lactation, menstruation, any acute or chronic infections, use of any medications, smoking, alcohol consumption, dieting or weight loss over last year, and calorie intake between $800-4200 \mathrm{kcal}$ per day. This study was approved by the Medical Research Ethics Committee of TUMS(IR.TUMS.MEDICINE.REC.1399.165).

\section{Anthropometric measures}

The weight of the participants was measured using a digital scale (Seca725 GmbH\& Co., Hamburg, Germany) and a precision of $0.1 \mathrm{~kg}$ without heavy clothing and shoes, and their height was measured by a rigid meter with a standing gauge, without shoes and hairpins with a precision of $0.5 \mathrm{~cm}$. The BMI was then calculated from the formula "weight divided by the square of the height». The waist was determined at the narrowest point between the lower ribs and the iliac crest, with light clothing and with a tape measure without putting pressure on the body, with an accuracy of about $0.5 \mathrm{~cm}$. To measure the weight to hip ratio(WHR), the waist is divided by and height, WHtR is calculated by dividing the waist by height.

\section{Dietary intake assessment}

Normal daily intakes were measured using the FFQ-147 item questionnaire previously validated and reliable FFQ contains a list of standard-sized foods that Iranians typically use[25]. Participants were asked to each food item over the past year that these amounts were first converted to daily intake and then to gram using household scales[26]. Dietary intakes were analyzed using Nutritionist 4(version 7.0; N-Squared Computing, Salem, OR).

\section{NRF index calculation}


The NRF Index offers a valid measure of the nutrient density of a person's diet[27,28]. This index has two parts: Nutrient-rich(NR) sector, which is based on a changed number of useful nutrients, limiting nutrients(LIM) sector. The NRF9.3 score is based on the sum of the percentages of reference daily values(DVs) of 9 beneficial nutrient proteins, dietary fiber, vitamin A, vitamin C, vitamin E, calcium, magnesium, iron, and potassium [29]. The daily reference values, shown in Table 2, are based on FDA standards[34,35]. which the selection of nutrients to persuasion, conforms the FDA explanation of healthy foods[30]), minus the entirety of the percentage of maximum suggested values for the 3 nutrients that are limited[29]. By the residual method, all the components we mentioned are adjusted with energy. We can use this index as follows: First, ENRF 9.3 algorithms based on the Dronovsky method score all foods eaten by each individual. [33], This results in an ENRF9.3 score (per 100 kcal) for each food item, meaning a food score of ENRF9.3. (see Table 1)[19].

Therefore, participants with a higher ENRF index score have a healthier dietary pattern than individuals with a lower ENRF index score.

Table1. Algorithms for ENRF index score and the NRn and LIM subscores, computed per 100 kcal

\begin{tabular}{|c|c|c|c|}
\hline Model & Algorithm & Reference value & Explanation \\
\hline $\begin{array}{l}\text { Subscores NRn } \\
\text { NRn_100kcal }\end{array}$ & $\begin{array}{l}\sum 1-n \text { (nutrient } \\
\left./ \mathrm{DV}_{\mathrm{i}}\right) / \mathrm{ED} \times 100\end{array}$ & $100 \mathrm{kcal}$ & $\begin{array}{l}\text { nutrient } \mathrm{i} \\
=\text { nutrient content } \mathrm{i} \text { in } 100 \\
- \text { g edible quota; } \\
\mathrm{DV}_{\mathrm{i}} \\
=\text { daily values for nutrient } \mathrm{i} \text { ( Table } \\
\mathrm{n}=\text { number of nutrients } \\
\mathrm{ED}=\text { energy density }\end{array}$ \\
\hline $\begin{array}{l}\text { Subscores LIM } \\
\qquad \text { LIM_100kcal }\end{array}$ & $\begin{array}{l}\sum 1-3\left(\mathrm{~L}_{i} / \mathrm{MRV}_{\mathrm{i}}\right) \\
/ \mathrm{ED} \times 100\end{array}$ & $100 \mathrm{kcal}$ & $\begin{array}{l}L_{\mathrm{i}}=\text { limiter nutrient } \mathrm{i} \text { content per } \\
100 \text { grams of the oral share of food, } \\
\mathrm{I}=1 \text { to } 3 ; \mathrm{ED}=\text { energy density }\end{array}$ \\
\hline compound models, & & $100 \mathrm{kcal}$ & discrepancy between sums \\
\hline $\mathrm{NRF}$ & NRn_100kcal & & \\
\hline NRFn.3_100kcal & LIM_100kcal & & \\
\hline
\end{tabular}

$\mathrm{MRV}=$ maximum recommended value; $\mathrm{NRF}=$ nutrient rich food; $\mathrm{LIM}=$ limited nutrient score.

Table 2. Daily reference values and maximum recommended nutrient values based on the $2000-$ kcal diet $^{1}$ 


\begin{tabular}{|lll|}
\hline Nutrient & RDV & MRV \\
\hline Fiber (g) & 25 & - \\
\hline Protein (g) & 50 & - \\
\hline Vitamin A (IU) & 5000 & - \\
\hline Vitamin C (mg) & 60 & - \\
\hline Vitamin E [IU (mg)] & $30(20)$ & - \\
\hline Iron (mg) & 18 & - \\
\hline Calcium (mg) & 1000 & - \\
\hline Potassium (mg) & 3500 & - \\
\hline Magnesium (mg) & 400 & - \\
\hline Saturated fat (g) & - & 20 \\
\hline Sodium (mg) & - & 2400 \\
\hline Sugars, added (g) & - & 50 \\
\hline
\end{tabular}

${ }^{1} \mathrm{RDV}$, reference daily value; MRV, maximum recommended value.

\section{Mental health assessment}

The mental status of the subjects was assessed using a validated questionnaire with 21 questions )DASS-21) [36]. This questionnaire measures the mood states of depression, stress, and anxiety. Each state has 7 items that are rated in a range of 4 points. Participants were asked by a trained dietitian to indicate how much each item was correct about them during the last week. A higher score indicates more severe mental distress. For the stress subgroup, the range of 15 to 18 is considered mild, 19 to 25 moderate, 26 to 33 severe, and $\geq 34$ very severe. Mild depression was 10-13, moderate was $14-20$, severe was $21-27$, and very severe was 28 . Mild, moderate, severe, and very severe anxiety were also considered 8-9, 10-14, 15-19, and $\geq 20$ respectively.

\section{Other variables}

To evaluate physical activity during the last 7 days, the valid and reliable Persian version of the International Physical Activity Questionnaire(IPAQ) was used[37]. Then the activity was converted into a daily metabolic equivalent (MET)-min per week. Researchers received demographic characteristics by questionnaire.

\section{Statistical analysis}

Analyzes were performed on 124 women. Kolmogorov-Smirnov method was used to assess the normality of the population. Quantitative variables across the ENRF 9.3 tertiles were shown as mean \pm standard deviations (SDs), and discrete variables were also expressed in numbers and percentages. analysis of variance (ANOVA) used for quantitative variables and Chi-square test for qualitative ones confounders such as age, $\mathrm{BMI}$ and physical activity, energy intake were adjusted by analysis of covariance (ANCOVA). The Bonferroni method is used for posthoc analyses. Multi-nominal logistic regression is used to compute odds ratio(OR) and $95 \% \mathrm{Cls}$, for the 
assessment association of the total DASS and its subgroups scores with the ENRF index. There was a significant difference when it was $p \leq 0.05$. Data were analyzed using IBM SPSS version 25.0 (SPSS, Chicago, IL, USA).

\section{Results}

In this cross-sectional study, 124 individuals were eligible for inclusion in the study. The mean ( $\pm S D)$ age, BMI, WHR, of individuals were $35.19 \pm 8.28$ (years) $30.48 \pm 4.22(\mathrm{~kg} / \mathrm{m} 2), 0.81 \pm 0.07$ respectively. $20 \%(\mathrm{n}=25)$ of participants were married. $1 \%(n=2)$ of study population were illiterate and $60 \%(n=75)$ of women owned-housing and $54 \%(n=68)$ of them took supplements. The percentage of $d$ depression in participating women was $44.4 \%$. The stress percentage was $53.2 \%$ and $55.6 \%$ of the study population had Anxiety.

\section{Association between quantitative and qualitative variables among ENRF9.3 index quartiles}

All participants were categorized based on the ENRF9.3 and divided into quartiles group. Table 1 demonstrates the general characteristics of subjects and quantitative and qualitative of the study participants across quartiles of ENRF9.3. Subjects with the highest ENRF9.3 index scores were older, even after controlling for confounders $(\mathrm{P}<0.05)$. Participants with a high ENRF9.3 score had significantly lower hip circumference and WHR after adjusting for age, $\mathrm{BMI}$, and physical activity ( $\mathrm{P}=0.04$ and $\mathrm{P}=0.05$ respectively). People who have more adherence to ENRF9.3 scores were single and homeowners $(P<0.05)$. Physical activity Mean in the highest quartiles was more than the lowest quartiles, which was significant at first $(P=0.05)$, but after adjustment showed marginal significance $(P=0.06)$. Regarding other variables related to general characteristics in ENRF9.3 quartiles, there was not a significant difference $(P>0.05)$ (Table 1).

Table1 Characteristics (quantitative and qualitative variables) of obese and overweight women among quartiles (Q) categories of NRF93 index. 


\begin{tabular}{|c|c|c|c|c|c|}
\hline \multicolumn{5}{|c|}{ Quartiles (Q) categories of NRF9.3 index } & \multirow{3}{*}{ Variables } \\
\hline P-value ${ }^{c}$ & Q4 & Q3 & Q2 & Q1 & \\
\hline \multirow[t]{2}{*}{$(P$-value *) } & $\mathrm{N}=31$ & $\mathrm{~N}=31$ & $\mathrm{~N}=31$ & $\mathrm{~N}=31$ & \\
\hline & & & & & $\begin{array}{l}\text { Quantitative } \\
\text { variables }^{\mathrm{a}}\end{array}$ \\
\hline $0.001(0.01)$ & $37.08 \pm 1.58$ & $38.22 \pm 1.55 \otimes$ & $31.43 \pm 1.61 \rrbracket$ & $33.27 \pm 1.66$ & Age(years) \\
\hline $0.1(0.8)$ & $160.57 \pm 1.53$ & $160.01 \pm 1.96$ & $162.60 \pm 2.17$ & $161.89 \pm 2.14$ & Height(cm) \\
\hline $0.4(0.7)$ & $78.41 \pm 1.53$ & $77.61 \pm 1.96$ & $80.65 \pm 2.17$ & $79.42 \pm 2.14$ & Weight(kg) \\
\hline$(0.06) 0.05$ & $865.3 \pm 0209.48$ & $1440.7 \pm 8203.57$ & $906.36 \pm 217.15$ & $630.50 \pm 216.81$ & PA (METs h/week) \\
\hline $0.8(0.5)$ & $29.80 \pm 0.82$ & $31.19 \pm 0.82$ & $29.91 \pm 0.85$ & $31.05 \pm 0.86$ & $\mathrm{BMI}\left(\mathrm{kg} / \mathrm{m}^{2}\right)$ \\
\hline $0.6(0.1)$ & $89.45 \pm 1.74$ & $91.71 \pm 2.22$ & $95.90 \pm 2.46$ & $94.46 \pm 2.43$ & Waist(cm) \\
\hline $0.7(0.04)$ & $113.91 \pm 1.02$ & $111.14 \pm 1.30$ & $109.89 \pm 1.44$ & $114.76 \pm 1.43$ & $\mathrm{Hip}(\mathrm{cm})$ \\
\hline $0.1(0.05)$ & $0.78 \pm 0.01$ & $0.82 \pm 0.02$ & $0.87 \pm 0.02$ & $0.82 \pm 0.02$ & WHR \\
\hline \multirow[t]{3}{*}{$0.6(0.3)$} & $0.55 \pm 0.01$ & $0.57 \pm 0.01$ & $0.59 \pm 0.01$ & $0.58 \pm 0.01$ & WHTR \\
\hline & & & & & $\begin{array}{l}\text { Qualitative } \\
\text { variables }{ }^{\text {b }}\end{array}$ \\
\hline & & & & & Marital status \\
\hline \multirow[t]{2}{*}{$0.007(0.04)$} & $9(36)$ & $0(0)$ & $6(24)$ & $10(40)$ & Married \\
\hline & $22(22.2)$ & $31(31.3)$ & $25(25.3)$ & $21(21.2)$ & Single \\
\hline \multirow[t]{5}{*}{$0.3(0.1)$} & & & & & Education \\
\hline & $0(0)$ & $0(0)$ & $2(100)$ & $0(0)$ & Illiterate \\
\hline & $6(35.3)$ & $5(29.4)$ & $4(23.5)$ & $2(11.8)$ & Undergraduate \\
\hline & $6(18.8)$ & $10(31.3)$ & $7(21.9)$ & $9(28.1)$ & Diploma \\
\hline & $19(26)$ & 16(21.9) & 18(24.7) & $20(27.4)$ & Master and upper \\
\hline $0.02(0.01)$ & $24(32)$ & $21(28)$ & $17(22.7)$ & 13(17.3) & $\begin{array}{l}\text { Housing } \\
\text { ownership }\end{array}$ \\
\hline $0.7(0.6)$ & 19(27.9) & $18(26.5)$ & $15(22.1)$ & $16(23.5)$ & $\begin{array}{l}\text { Supplement } \\
\text { intake }\end{array}$ \\
\hline \multirow[t]{2}{*}{$0.9(0.4)$} & $12(25.5)$ & 10(21.3) & $12(25.5)$ & 13(27.7) & $\begin{array}{l}\text { Job } \\
\text { status(Employed) }\end{array}$ \\
\hline & & & & & Economic status \\
\hline \multirow[t]{2}{*}{$0.2(0.08)$} & 10030.3 & $7(21.2)$ & 10(30.3) & $6(18.2)$ & Poor level \\
\hline & 12(19.7) & $15(24.6)$ & 12(19.7) & $22(36.1)$ & Moderate level \\
\hline
\end{tabular}


NRF, nutrient-rich food; PA, physical activity; BMI, body mass index; WHR, waist to hip ratio; WHTR, waist to height ratio

${ }^{a}$ means $\pm S D$ (standard deviation); $\otimes n(\%) ; ~ \otimes P$-value result from ANOVA for quantitative variables and Chi-square test for qualitative variables and * $p$-value result from the analysis of covariance test (ANCOVA) adjusted for age, $\mathrm{BMI}$ and physical activity; $\mathbb{\nabla}$ association quartile 2 and 3

The Association of ENRF9.3 index components, micronutrient, macronutrient, and food groups across the ENRF9.3 index score quartiles

The relation of food items consumed across the ENRF9.3 index quartiles is shown in Table 2. participants who were in the first quartile of ENRF9.3 index had lower intakes of vitamin $A(P<0.001)$, Potassium $(P=0.03)$, and calcium $(P=0.05)$ and higher intakes of vitamin $E(P=0.01)$. There was no significant difference for other dietary intakes $(P>0.05)$. After adjusting for energy intake, a significant relationship has been seen in protein intake, and magnesium among ENRF9.3 quartiles, so that their mean was higher in the upper quartile, but the others remained insignificant $(P>0.05)$. Among food groups in the crude model, vegetables, milk and milk products, lowfat dairy, vegetable oil, meat and poultry, processed meat and tea and coffee intake was higher in the highest quartile of ENRF9.3 score, which was also significant $(P<0.05)$. But, no significant association was observed for the other food groups $(P>0.05)$. After controlling energy intake, a lower mean of $z i n c(P=0.01)$, niacin $(P=0.002)$, beta carotene, riboflavin, pyridoxine, folate, biotin, and copper $(P<0.001)$ consumption, and significantly higher MUFA $(P=<0.001)$, PUFA, and linoleic $(P=0.002)$ consumption, were observed at the highest ENFR9.3 quartile relative to the lowest, while there was no significant difference for another dietary intake $(P>0.05)$.

Table2 ENRF9.3 index score and its components, food groups, macronutrients, and micronutrients among quartiles (Q) categories of ENRF9.3 index 


\begin{tabular}{|c|c|c|c|c|c|}
\hline \multirow{3}{*}{ Variables } & \multicolumn{5}{|c|}{ Quartiles (Q) of ENRF9.3 index } \\
\hline & Q1 & Q2 & Q3 & Q4 & \multirow{2}{*}{$\begin{array}{l}\text { P- } \\
\text { value }^{a}\end{array}$} \\
\hline & \multicolumn{4}{|l|}{ Mean $\pm S D$} & \\
\hline NRF9.3 index score & $55.25 \pm 1.95$ & $69.17 \pm 2.02$ & $81.15 \pm 2.02$ & $106.94 \pm 2.08$ & $<0.001$ \\
\hline Model & $55.27 \pm 1.95$ & $69.03 \pm 2.02$ & $81.40 \pm 2.03$ & $106.81 \pm 2.09$ & $<0.001$ \\
\hline NRF9 index score & $81.88 \pm 1.09$ & $89.80 \pm 1.12$ & $94.22 \pm 1.12$ & $102.10 \pm 1.16$ & $<0.001$ \\
\hline Model 1 & $81.88 \pm 1.09$ & $89.82 \pm 1.13$ & $94.19 \pm 1.13$ & $102.12 \pm 1.17$ & $<0.001$ \\
\hline \multicolumn{6}{|l|}{$\begin{array}{l}\text { NRF9 index } \\
\text { components }\end{array}$} \\
\hline Protein(gr/100kcal) & $82.50 \pm 4.32$ & $86.38 \pm 4.46$ & $97.52 \pm 4.46$ & $86.72 \pm 4.61$ & 0.1 \\
\hline Model 1 & $82.17 \pm 2.63$ & $88.95 \pm 2.72$ & $93.07 \pm 2.73$ & $89.11 \pm 2.81$ & 0.03 \\
\hline $\begin{array}{l}\text { Total } \\
\text { fiber(g/100kcal) }\end{array}$ & $49.35 \pm 3.04$ & $45.67 \pm 3.14$ & $44.37 \pm 3.14$ & $43.16 \pm 3.25$ & 0.5 \\
\hline Model 1 & $49.18 \pm 2.49$ & $46.99 \pm 2.57$ & $42.08 \pm 2.59$ & $44.39 \pm 2.66$ & 0.2 \\
\hline Vitamin A(IU) & $1848.23 \pm 188.95$ & $2426.35 \pm 194.95$ & $3066.26 \pm 194.95$ & $2405.74 \pm 201.56$ & $<0.001$ \\
\hline Model & $1839.54 \pm 167.38$ & $2492.77 \pm 173.06$ & $2951.30 \pm 173.81$ & $2467.52 \pm 178.86$ & $<0.001$ \\
\hline Vitamin C(mg) & $192.67 \pm 23.28$ & $178.45 \pm 24.02$ & $198.59 \pm 24.02$ & $160.15 \pm 24.84$ & 0.6 \\
\hline Model 1 & $191.76 \pm 21.43$ & $185.40 \pm 22.16$ & $186.55 \pm 22.25$ & $166.62 \pm 22.90$ & 0.8 \\
\hline Vitamin $\mathrm{E}(\mathrm{mg})$ & $24.52 \pm 1.86$ & $17.84 \pm 1.92$ & $18.27 \pm 1.92$ & $16.17 \pm 1.98$ & 0.01 \\
\hline Model 1 & $17.47 \pm 1.81$ & $18.19 \pm 1.87$ & $24.66 \pm 1.88$ & $16.50 \pm 1.93$ & 0.01 \\
\hline Calcium(mg) & $1041.58 \pm 65.90$ & $1166.68 \pm 67.99$ & $1303.10 \pm 67.99$ & $1209.16 \pm 70.30$ & 0.05 \\
\hline Model 1 & $1037.02 \pm 46.59$ & $1201.60 \pm 48.17$ & $1242.65 \pm 48.38$ & $1241.65 \pm 49.78$ & 0.007 \\
\hline Magnesium (mg) & $437.88 \pm 22.18$ & $422.99 \pm 22.89$ & $495.53 \pm 22.89$ & $461.57 \pm 23.66$ & 0.1 \\
\hline Model 1 & $436.09 \pm 12.39$ & $436.75 \pm 12.81$ & $471.71 \pm 12.87$ & $474.37 \pm 13.24$ & 0.04 \\
\hline Potassium (mg) & $3702.33 \pm 249.10$ & $4039.57 \pm 257.01$ & $4694.69 \pm 257.01$ & $4405.30 \pm 265.72$ & 0.03 \\
\hline Model 1 & $3683.47 \pm 157.74$ & $4183.84 \pm 163.09$ & $4444.96 \pm 163.80$ & $4539.50 \pm 168.55$ & 0.001 \\
\hline Iron(mg) & $19.17 \pm 0.87$ & $18.43 \pm 0.90$ & $19.77 \pm 0.90$ & $17.70 \pm 0.93$ & 0.4 \\
\hline Model 1 & $19.10 \pm 0.48$ & $18.97 \pm 0.49$ & $18.83 \pm 0.50$ & $18.21 \pm 0.51$ & 0.6 \\
\hline
\end{tabular}


LIM3 index score $\quad 21.19 \pm 0.58$

Model $1 \quad 16.19 \pm 0.58$
$18.14 \pm 0.60$

$18.15 \pm 0.60$
$18.84 \pm 0.60$

$18.83 \pm 0.60$
$16.34 \pm 0.62$

$21.35 \pm 0.62$
$<0.001$

$<0.001$

\section{LIM3 index components}

\begin{tabular}{|c|c|c|c|c|c|}
\hline Sodium(mg) & $4328.65 \pm 239.30$ & $4221.80 \pm 246.90$ & $4578.50 \pm 246.90$ & $4262.16 \pm 255.27$ & 0.7 \\
\hline Model & $4314.85 \pm 193.86$ & $4327.32 \pm 200.45$ & $4395.84 \pm 201.31$ & $4360.31 \pm 207.16$ & 0.9 \\
\hline Saturated fat $(\mathrm{g})$ & $27.34 \pm 1.91$ & $27.38 \pm 1.97$ & $31.82 \pm 1.97$ & $28.64 \pm 2.04$ & 0.3 \\
\hline Model & $27.20 \pm 1.28$ & $28.45 \pm 1.32$ & $29.97 \pm 1.33$ & $29.63 \pm 1.37$ & 0.4 \\
\hline Add sugar(g) & $15.20 \pm 2.86$ & $15.07 \pm 2.95$ & $21.66 \pm 2.95$ & $17.01 \pm 3.05$ & 0.3 \\
\hline Model & $15.12 \pm 2.76$ & $15.66 \pm 2.85$ & $20.63 \pm 2.86$ & $17.56 \pm 2.95$ & 0.5 \\
\hline
\end{tabular}

\section{Food groups}

Grain

Whole grains(g)

$\begin{array}{lllllll} & \text { Crude } & 518.93 \pm 38.34 & 478.23 \pm 38.98 & 500.23 \pm 38.98 & 513.70 \pm 38.34 & 0.8 \\ 2 & \text { Model } & 473.82 \pm 41.68 & 450.44 \pm 44.00 & 484.68 \pm 42.19 & 544.47 \pm 43.19 & 0.4\end{array}$

Refined grains(g)

$\begin{array}{lllllll} & \text { Crude } & 425.45 \pm 38.11 & 390.77 \pm 38.74 & 404.76 \pm 38.74 & 434.65 \pm 38.11 & 0.8 \\ 2 & \text { Model } & 380.06 \pm 41.86 & 365.78 \pm 44.19 & 392.82 \pm 42.37 & 455.72 \pm 43.37 & 0.4\end{array}$

Vegetables(g)

$\begin{array}{rrrrrrr} & \text { Crude } & 271.96 \pm 35.80 & 380.24 \pm 36.39 & 504.51 \pm 36.39 & 413.84 \pm 35.80 & <0.001 \\ 2 & \text { Model } & 313.07 \pm 41.86 & 388.62 \pm 44.19 & 493.23 \pm 42.38 & 408.47 \pm 43.38 & 0.03\end{array}$

Fruits(g)

$\begin{array}{cccccc}\text { Crude } & 441.41 \pm 57.40 & 488.57 \pm 58.35 & 555.71 \pm 58.35 & 528.49 \pm 57.40 & 0.5 \\ \text { Model2 } & 450.40 \pm 58.33 & 446.09 \pm 61.58 & 528.71 \pm 59.05 & 493.31 \pm 60.44 & 0.7\end{array}$

Legumes(g)

\begin{tabular}{llllll} 
Crude & $55.76 \pm 7.04$ & $57.48 \pm 7.26$ & $57.30 \pm 7.26$ & $45.88 \pm 7.51$ & 0.8 \\
\hline Model 2 & $60.02 \pm 7.88$ & $52.54 \pm 8.31$ & $52.01 \pm 7.97$ & $50.28 \pm 8.16$ & 0.8
\end{tabular}

Nut and seeds(g)

Crude

$11.56 \pm 2.63$

$12.54 \pm 2.71$

$12.68 \pm 2.71$

$14.97 \pm 2.81$

0.9 


$\begin{array}{llllll}\text { Model } 2 & 13.39 \pm 3.20 & 11.91 \pm 3.38 & 11.20 \pm 3.24 & 17.00 \pm 3.31 & 0.6\end{array}$

Milk and milk

products(g)

2

\begin{tabular}{llllll} 
Crude & $243.62 \pm 36.72$ & $364.83 \pm 37.33$ & $427.77 \pm 37.33$ & $410.05 \pm 36.72$ & 0.003 \\
\hline Model & $271.83 \pm 42.64$ & $320.41 \pm 45.02$ & $448.17 \pm 43.17$ & $428.08 \pm 44.19$ & 0.01
\end{tabular}

Low-fat dairy(g)

$\begin{array}{rrrrrrr} & \text { Crude } & 191.03 \pm 32.14 & 289.06 \pm 32.68 & 324.11 \pm 32.68 & 300.96 \pm 32.14 & 0.02 \\ 2 & \text { Model } & 207.79 \pm 37.48 & 266.73 \pm 39.57 & 327.20 \pm 37.94 & 318.36 \pm 38.84 & 0.1\end{array}$

Fats and oils(g)

$\begin{array}{lllllll} & \text { Crude } & 49.13 \pm 4.91 & 36.52 \pm 4.99 & 35.87 \pm 4.99 & 34.61 \pm 4.91 & 0.1 \\ 2 & \text { Model } & 49.95 \pm 5.44 & 33.24 \pm 5.74 & 34.37 \pm 5.51 & 39.83 \pm 5.64 & 0.1\end{array}$

Vegetable oil(g)

Model2

Crude

$39.26 \pm 4.51$

$24.86 \pm 4.58$

$23.14 \pm 4.58$

$23.71 \pm 4.51$

0.03

$40.95 \pm 4.98$

$23.97 \pm 5.26$

$22.05 \pm 5.04$

$28.40 \pm 5.16$

0.04

$\operatorname{Eggs}(\mathrm{g})$

2

\begin{tabular}{llllll} 
Crude & $18.80 \pm 2.68$ & $18.78 \pm 2.72$ & $23.41 \pm 2.72$ & $21.68 \pm 2.68$ & 0.5 \\
\hline Model & $17.83 \pm 2.96$ & $17.74 \pm 3.13$ & $22.90 \pm 3.00$ & $23.95 \pm 3.07$ & 0.3
\end{tabular}

Meat and

poultry(g)

2

$\begin{array}{rllllll} & \text { Crude } & 45.60 \pm 6.70 & 56.03 \pm 6.81 & 76.20 \pm 6.81 & 55.69 \pm 6.70 & 0.01 \\ 2 & \text { Model } & 45.42 \pm 7.84 & 53.37 \pm 8.28 & 77.31 \pm 7.94 & 61.26 \pm 8.13 & 0.04\end{array}$

2

Processed

meat(g)

Fish and seafood

(g)

\begin{tabular}{|c|c|c|c|c|c|c|}
\hline & Crude & $7.93 \pm 1.95$ & $9.51 \pm 1.98$ & $10.88 \pm 1.98$ & $11.88 \pm 1.95$ & 0.5 \\
\hline 2 & Model & $7.77 \pm 2.32$ & $9.04 \pm 2.45$ & $11.34 \pm 2.35$ & $14.14 \pm 2.41$ & 0.2 \\
\hline
\end{tabular}


Sugar, sweets, and

beverage(g)

Model2

Crude

$21.54 \pm 9.09$

$25.96 \pm 9.24$

$42.10 \pm 9.24$

$15.91 \pm 9.09$

0.2

$22.42 \pm 10.92$

$25.15 \pm 11.53$

$47.73 \pm 11.06$

$16.98 \pm 11.32$

0.2

Tea and coffee

Crude

Model2

$349.79 \pm 65.77 \quad 443.89 \pm 66.86$

$699.77 \pm 66.86$

$1196.88 \pm 65.77$

$<0.001$

$431.99 \pm 70.79$

$422.93 \pm 74.73$

$657.96 \pm 71.66$

$1200.67 \pm 73.35$

$<0.001$

\section{Dietary intake}

Energy (kcal)

Energy from fat (\%)

Energy from

protein $(\%)$

Energy from
carbohydrate (\%)

Zinc (mg)

Copper (mg)

Selenium (mcg)

Beta carotene $(\mathrm{Mg}) \quad 6658.55 \pm 382.16$

Thiamin (mg)

Niacin (mg)

Riboflavin (mg)

Pyridoxine $(\mathrm{mg})$

Cobalamin(mcg)

Folate $(\mathrm{mcg})$

Biotin (mg)

MUFA (g)

PUFA $(\mathrm{g})$

Linoleic acid(mg)

Linolenic acid $(\mathrm{mg}) \quad 1.08 \pm 0.10$

EPA(mg)

DHA(mg)

Caffeine (mg)
$2703.25 \pm 593.05$

$28.34 \pm 1.06$

$14.09 \pm 0.39$

$61.06 \pm 1.02$

$13.57 \pm 0.36$

$2.23 \pm 0.05$

$124.43 \pm 4.41$

$130.25 \pm 4.41$

$5442.31 \pm 382.34$

$2.18 \pm 0.06$

$25.43 \pm 0.80$

$2.30 \pm 0.09$

$2.15 \pm 0.06$

$4.48 \pm 0.35$

$625.14 \pm 16.63$

$38.68 \pm 1.67$

$30.19 \pm 1.55$

$19.16 \pm 1.46$

$16.61 \pm 1.45$

$1.23 \pm 0.10$

$0.02 \pm 0.006$

$0.09 \pm 0.01$

$148.91 \pm 16.54$

Page $12 / 22$

$0.10 \pm 0.01$

$125.89 \pm 16.53$
$2822.42 \pm 673.68 \quad 2568.83 \pm 740.52 \quad 0.4$

$34.99 \pm 1.07$

$37.28 \pm 1.06$

$<0.001$

$13.56 \pm 0.39$

$12.13 \pm 0.39$

0.005

$53.90 \pm 1.03$

$52.74 \pm 1.02$

$<0.001$

$12.59 \pm 0.37$

$12.12 \pm 0.36$

0.01

$1.84 \pm 0.05$

$1.69 \pm 0.05$

$<0.001$

$124.00 \pm 4.48$

$120.23 \pm 4.41$

0.4

$4122.67 \pm 388.21 \quad 3258.33 \pm 381.95<0.001$

$2.12 \pm 0.06$

$2.02 \pm 0.06$

0.1

$25.38 \pm 0.81$

$22.25 \pm 0.80$

0.002

$2.12 \pm 0.09$

$1.90 \pm 0.09$

$<0.001$

$2.04 \pm 0.06$

$1.82 \pm 0.06$ $<0.001$

$4.25 \pm 0.35$

$3.83 \pm 0.35$

0.4

$601.74 \pm 16.89$

$553.40 \pm 16.61$

$<0.001$

$34.56 \pm 1.70$

$29.18 \pm 1.67$

$<0.001$

$36.28 \pm 1.57$

$38.24 \pm 1.54$

$<0.001$

$24.47 \pm 1.48$

$24.21 \pm 1.46$

0.002

$21.77 \pm 1.47$

$21.71 \pm 1.45$

0.002

$1.23 \pm 0.10$

$1.44 \pm 0.10$

0.1

$0.02 \pm 0.006$

$0.08 \pm 0.01$

$0.02 \pm 0.006$

0.7

$116.81 \pm 16.79$

$0.08 \pm 0.01$

0.7 0.08 
NRF9.3, nutrient-rich food; NRF9, nutrient-rich; LIM3, nutrients to limit; MUFA, monounsaturated fatty acids; PUFA, polyunsaturated fatty acids; EPA, Eicosapentaenoic acid; DHA, Docosahexaenoic acid

NRF9.3 components model 1 are mean \pm standard error, adjust for energy, Food groups model 2are mean \pm standard error, adjust for age, BMI, physical activity, obtained from the analysis of covariance (ANCOVA), Macronutrient and micronutrient are mean \pm standard error, adjust for energy intake, obtained from the analysis of covariance. Other variables are mean \pm standard deviation, one-way analysis of variance was used for comparison of all variables among quartile of NRF9.3 score.

The ap-values result from the ANOVA test for the crude model and analysis of covariance test(ANCOVA) for the adjusted model.

\section{DASS total score and its subgroups among quartiles of the ENRF9.3 index score}

In Table 3, the DASS total score and its subgroups among ENRF9.3 index score quartiles assessed, so that the mean of total DASS score was lower in higher quartiles, there was a significant relationship $(P=0.05)$, which change to marginal significance after adjustment with potential confounders in model 1 (age and energy intake) $(P=0.07)$, lost its significance with further adjustment with confounders in model 2and model 3 and $4(P=0.08)$. All of the three-component of mental health stress, anxiety, and depression and total DASS score was lower in the top quartile. The stress of individuals, in the crude model, was marginally significant $(\mathrm{P}=0.06)$, and after adjustment for potential confounders in models 2,3 , and 4 was significant $(P=0.04)$ which according to Bonferroni analysis, this significant difference was observed between the means of quartile 4 and 1 so that the mean is lower in quartile 4 . There were no significant differences in terms of depression and anxiety after adjustment ( $P>0.05)$. ) Table 3$)$

Table3 DASS total score and subscales among quartiles (Q) categories of ENRF9.3 index in overweight and obese women 


\begin{tabular}{|c|c|c|c|c|c|c|}
\hline \multicolumn{5}{|c|}{ Quartiles (Q) categories of NRF9.3 index } & \multirow{2}{*}{$\begin{array}{l}\text { Model } \\
{[}\end{array}$} & \multirow[t]{2}{*}{ Variables } \\
\hline P-valuec & Q4 & Q3 & Q2 & Q1 & & \\
\hline 0.05 & $25.90 \pm 5.07$ & $35.61 \pm 4.27$ & $34.25 \pm 4.27$ & $43.35 \pm 3.76$ & Crude & \multirow{5}{*}{$\begin{array}{l}\text { DASS-21 total } \\
\text { Score }\end{array}$} \\
\hline 0.07 & $33.68 \pm 4.63$ & $34.67 \pm 4.51$ & $34.98 \pm 4.53$ & $40.40 \pm 4.30$ & 1 & \\
\hline 0.08 & $33.96 \pm 5.23$ & $35.53 \pm 5.01$ & $30.13 \pm 5.15$ & $41.47 \pm 4.86$ & 2 & \\
\hline 0.08 & $30.43 \pm 6.69$ & $35.42 \pm 4.93$ & $31.98 \pm 5.33$ & $40.87 \pm 5.34$ & 3 & \\
\hline 0.08 & $30.44 \pm 6.74$ & $35.43 \pm 4.97$ & $31.96 \pm 5.40$ & $40.87 \pm 5.37$ & 4 & \\
\hline 0.1 & $6.90 \pm 2.05$ & $11.09 \pm 1.73$ & $9.61 \pm 1.73$ & $12.40 \pm 1.52$ & Crude & \multirow{5}{*}{$\begin{array}{l}\text { DASS-21 depression } \\
\text { subscale } \\
\text { score }\end{array}$} \\
\hline 0.8 & $9.29 \pm 1.85$ & $10.43 \pm 1.80$ & $10.25 \pm 1.81$ & $11.48 \pm 1.72$ & 1 & \\
\hline 0.4 & $9.29 \pm 2.14$ & $11.14 \pm 2.05$ & $7.85 \pm 2.11$ & $11.87 \pm 1.99$ & 2 & \\
\hline 0.7 & $9.77 \pm 2.75$ & $11.07 \pm 2.03$ & $7.83 \pm 2.19$ & $10.65 \pm 2.19$ & 3 & \\
\hline 0.1 & $9.81 \pm 2.77$ & $11.10 \pm 2.04$ & $7.76 \pm 2.22$ & $10.63 \pm 2.21$ & 4 & \\
\hline 0.1 & $7.27 \pm 1.60$ & $9.16 \pm 1.35$ & $8.90 \pm 1.35$ & $11.80 \pm 1.19$ & Crude & \multirow{5}{*}{$\begin{array}{l}\text { DASS-21 anxiety } \\
\text { subscale score }\end{array}$} \\
\hline 0.4 & $8.74 \pm 1.45$ & $9.06 \pm 1.41$ & $8.98 \pm 1.42$ & $11.48 \pm 1.35$ & 1 & \\
\hline 0.3 & $8.19 \pm 1.62$ & $9.27 \pm 1.55$ & $7.47 \pm 1.59$ & $11.45 \pm 1.50$ & 2 & \\
\hline 0.4 & $7.70 \pm 2.10$ & $9.21 \pm 1.55$ & $7.79 \pm 1.68$ & $11.15 \pm 1.68$ & 3 & \\
\hline 0.4 & $7.58 \pm 2.11$ & $9.13 \pm 1.56$ & $7.96 \pm 1.69$ & $11.18 \pm 1.68$ & 4 & \\
\hline 0.06 & $11.72 \pm 2.21$ & $15.35 \pm 1.87$ & $15.74 \pm 1.87$ & $19.15 \pm 1.64$ & Crude & \multirow{5}{*}{$\begin{array}{l}\text { DASS-21 stress } \\
\text { Subscale } \\
\text { Score }\end{array}$} \\
\hline 0.4 & $15.65 \pm 2.01$ & $15.17 \pm 1.96$ & $15.74 \pm 1.97$ & $17.43 \pm 1.87$ & 1 & \\
\hline 0.04 & $16.48 \pm 2.24$ & $15.10 \pm 2.15$ & $14.81 \pm 2.21$ & $18.14 \pm 2.08$ & 2 & \\
\hline 0.04 & $12.95 \pm 2.85$ & $15.14 \pm 2.10$ & $16.35 \pm 2.27$ & $19.06 \pm 2.27$ & 3 & \\
\hline 0.04 & $\begin{array}{l}13.03 \pm 2.87 \\
\end{array}$ & $15.19 \pm 2.11$ & $16.23 \pm 2.30$ & $19.04 \pm 2.28$ & 4 & \\
\hline
\end{tabular}

DASS, depression anxiety stress score; NRF, nutrient-rich food ${ }^{\text {a }}$ mean $\pm S E$

Model1: adjusted for age and energy intake; model2: more adjusted for marital status, housing ownership, job status, family size, supplement intake, physical activity, and economic status; model3: more adjusted for the consumption of dietary linoleic acid; model4: more adjusted for BMI. $\otimes$ Association quartile 1 and $4 .{ }^{C}$ p-values result from the ANOVA test for the crude model and analysis of covariance test(ANCOVA) for the adjusted model.

\section{Associations between total DASS score and its subgroups with ENRF9.3 score}

The odds ratio(OR) and its $95 \% \mathrm{Cl}$ of total DASS score and its subgroups with ENRF9.3 score were represented in Table 4. In the crude model, the relation between only the mild depression subgroup and ENRF9.3 was 
significant( $\mathrm{OR}=0.97 ; 95 \% \mathrm{Cl} 0.94$ to $; \mathrm{P}=0.05)$ and showed a $0.03 \%$ decrease with increasing ENRF9.3 quartiles, however, there was no association between mild depression index with ENRF9.3 in the adjusted model(OR=0.98; $95 \% \mathrm{Cl}=0.95-1.01 ; \mathrm{P}=0.3)$. After controlling the confounders by multinomial logistic regression analysis, the other subgroups of the total DASS score also did not show a significant relation $(P>0.05)$.

Table 4 Relationship of DASS-21 scale and subscales with an NRF9.3 index score

\begin{tabular}{|c|c|c|c|c|c|c|}
\hline \multirow{2}{*}{ Variable } & \multicolumn{3}{|c|}{ Crude model } & \multicolumn{3}{|c|}{ Adjust model* } \\
\hline & $\mathrm{OR}$ & $0.95 \% \mathrm{Cl}$ & p-value & OR & $0.95 \% \mathrm{Cl}$ & p-value \\
\hline \multicolumn{7}{|l|}{ Mental health } \\
\hline \multicolumn{7}{|c|}{ DASS-21 stress subscaled } \\
\hline Normala $^{a}$ & _- & _ & _- & - & _- & - \\
\hline Mild & 0.97 & $0.94-1.00$ & 0.09 & 0.97 & $0.94-1.00$ & 0.1 \\
\hline Moderate & 1.00 & $0.98-1.02$ & 0.6 & 1.00 & $0.98-1.03$ & 0.5 \\
\hline Severe & 1.01 & $0.98-1.03$ & 0.3 & 1.02 & $0.99-1.05$ & 0.1 \\
\hline Extremely Severe & 0.97 & $0.94-1.01$ & 0.2 & 0.95 & $0.90-1.01$ & 0.1 \\
\hline \multicolumn{7}{|c|}{ DASS-21 depression Subscale] } \\
\hline Normal $^{a}$ & - & - & - & - & - & - \\
\hline Mild & 0.97 & $0.94-1.00$ & 0.05 & 0.98 & $0.95-1.01$ & 0.3 \\
\hline Moderate & 0.98 & $0.96-1.01$ & 0.3 & 0.98 & $0.95-1.01$ & 0.2 \\
\hline Severe & 1.00 & $0.97-1.03$ & 0.5 & 1.00 & $0.97-1.04$ & 0.6 \\
\hline Extremely Severe & 0.99 & $0.96-1.02$ & 0.6 & 0.99 & $0.95-1.02$ & 0.6 \\
\hline \multicolumn{7}{|c|}{ DASS-21 anxiety Subscale $\rrbracket$} \\
\hline Normala $^{a}$ & - & - & - & - & - & - \\
\hline Mild & 1.01 & $0.98-1.03$ & 0.3 & 0.99 & $0.96-1.02$ & 0.7 \\
\hline Moderate & 1.00 & $0.98-1.02$ & 0.6 & 1.00 & $0.97-1.02$ & 0.9 \\
\hline Severe & 0.99 & $0.96-1.02$ & 0.7 & 0.99 & $0.95-1.03$ & 0.7 \\
\hline Extremely Severe & 0.99 & 0.97-1.02 & 0.7 & 0.99 & $0.96-1.02$ & 0.8 \\
\hline
\end{tabular}

DASS, depression anxiety stress score; NRF, nutrient-rich food,

${ }^{a}$ As a reference group, $\otimes$ mild stress(15-18), moderate stress(19-25), severe stress(26-33), extremely severe $\operatorname{stress}(\geq 34)$. $\otimes$ mild depression(10-13), moderate depression(14-20), severe depression(21-27), extremely severe depression ( $\geq 28$ ). $\triangle$ mild anexity(8-9), moderate anexity(10-14), severe anexity(15-19), extremely severe anexity( $\geq 20$ )

P-valueresult from multinominal logestic regression test 


\section{Discussion}

In the present study, the mental health subgroups, such as the stress, anxiety, and depression, before and after adjustment for the confounders, were lower mean in the upper ENRF quartiles, but significance was observed only for the stress subgroup after controlling the confounders.

Diet plays an important role in improving or advancing mental illness In line with our study concerning depression and anxiety, Lizanne JS. Schweren and et al. in a cohort study showed that diet is unlikely to prevent depression and anxiety in the community in the long run[38] and Murakami et al. also in their systematic review, found no association between dietary variables and depressive symptoms[39]. However, against these studies, Poorrezaeian and colleagues in two cross-sectional study found an inverse relationship between anxiety and depression with dietary diversity score as the healthy eating index in women $[40,41]$, because dietary diversity is positively associated with dietary micronutrients and negatively correlated with micronutrient deficiencies intake[42], and the relationship between the consumption of processed foods, commercial baked goods, fast foods, and sweets, with an increased risk of depression, due to the high content of saturated fats, trans-fatty acids, added sugar and refined carbohydrates and low content of fiber and nutrients, has been seen in observational studies[43]. Gibson-Smith et al. found that the quality of the diet was poorer in people with anxiety disorders and depression[44]. Another opposed study has shown, people who received a Mediterranean diet in 3 months had better scores on depression and overall mental health scores than those who did not, due to improvements in the quality of their diet[45].

Deficiency in some B and C vitamins and iron has been linked to psychological symptoms[46], and many of the nutrients offered are effective against depression[47, 48], in this context, Lisa and colleagues report that folate, B12, iron, selenium, and zinc deficiency are more common in depressed people[49]. Because vitamin D affects brain structure, neuronal differentiation, binding, dopamine pathways, neurotransmitters, brain calcium homeostasis, inflammatory markers, nerve growth factor synthesis, and the response of the hypothalamicpituitary-adrenocortical axis to threat, zinc is important for energy metabolism, macronutrient stabilization, regulation of protein synthesis, neuronal progenitor cell activity, neurotransmitters, neutrophils, and antioxidants, iron is needed for energy production, phospholipid metabolism, myelination and the synthesis of neurotransmitters and DNA[50], calcium works by activating tryptophan hydroxylase and synthesizing serotonin[51] and omega-3 fatty acid has anti-inflammatory or neuroendocrine modulating effects[52]. Folatederived coenzymes, B12 and B6, are also involved in the synthesis and metabolism of dopamine and serotonin. Besides, vitamins B6 and B12, as a cofactor, convert homocysteine, that inadequate nutrition of these vitamins can lead to the accumulation of homocysteine and reduced monoamines synthesis in the brain, which may play a vital role in mental health etiology[53]. Therefore, perhaps the reason for not meaningful of anxiety and depression in the present study can be related to a significant reduction in B vitamins and zinc, and meaninglessness of nutrients such as vitamin D, omega 3, iron, and vitamin $C$ in quartiles of ENRF index score.

In our study, among all of the food groups, vegetables, dairy, meat and poultry, and tea and coffee were significant across ENRF quartiles. Micronutrients in vegetables like B group vitamins and their metabolites[5456] and the others such as vitamin C[57], calcium[58], and zinc[59], which regulate brain pathways [60]. green leafy vegetables are proper sources of magnesium that insufficient magnesium can cause headaches and 
fatigue and exacerbate the effects of stress[61]. In our study, magnesium intake also increased with increasing ENRF quartile, which can be one of the reasons for a significant stress reduction. Because magnesium is the main cofactor in the synthesis of neurotransmitters and adrenaline and is participated in neuronal cell metabolism[62]. It was seen that low serum magnesium concentrations, in response to stress, increase the release of stress-related hormones, including cortisol, adrenocorticotrophic hormone, and catecholamines[63, 64], which affects their access to the brain and creates a vicious cycle of reduced stress resistance and further magnesium depletion $[65,66]$. In addition to the vegetables, in the study of Laugero et al., stress was also associated with lower protein intake[67], because of the amino acid tryptophan in meat, which can play a momentous role in relieving mood, satiety, and sleep regulation by converting to serotonin that could help mental health[68]. The positive relationship between dairy consumption, with some cognitive and psychological health measures, has been found in the study of Crichton et al[69]. In our study, calcium was higher at the top of the ENRF quartile, since milk and other dairy products account for more than half of the dietary calcium intake in most parts of the world[70], their stress-relieving properties can also be attributed to more calcium intake. People with higher HEl scores are more likely to eat nutritious foods[71]that when replacing junk food with fresh, highfiber plant-based foods, they will consume few nutrients that are essential for healthy metabolism and provide significant stress protection [72]. Vitamin B is used as a cofactor for adrenaline synthesis in stress response, and it is also used to synthesize neurotransmitters such as serotonin and dopamine that affect stress[73, 74].

The present study is the first study that examines the relationship between ENRF index score and mental health can be considered as one of its strengths. However, there were some limitations, the cross-sectional design of the study, which makes the result can't find out the casualty, use the FFQ questionnaire to assess food intake, which depends on a person's memory, and restricting the gender of the study population, that decreased generalisability.

\section{Conclusions}

In this study, there was no significant relationship between overall DASS score, anxiety, and depression with the ENRF index score. There was a significant relationship between stress and ENRF, more studies are needed especially with a prospective plan.

\section{Abbreviations}

ENRF: energy adjusts Nutrient-rich foods, TUMS: Tehran University of Medical Science, DASS: Depression Anxiety Stress Scales, BMI: Body Mass Index, HEl: Healthy Eating Index, WHR: weight to hip ratio, WHTR: waist to height ratio, FFQ: Food Frequency Questionnaire, DVs: daily values, NRn: Nutrient Rich nutrient, MRV: maximum recommended value, NRF: nutrient-rich food; LIM: limited nutrient score, RDV: reference daily value, IPAQ: International Physical Activity Questionnaire, MET: metabolic equivalent, SDs: Standard Deviations, ANOVA: analysis of variance, ANCOVA: analysis of covariance, MUFA: monounsaturated fatty acids, PUFA: polyunsaturated fatty acids, EPA: Eicosapentaenoic acid, DHA: Docosahexaenoic acid, DNA: Dinocloic acid

\section{Declarations}

\section{Acknowledgment}

We thank all those involved and participants for taking part in the present study. 
Authors' contributions

ZS wrote the article, KhM revisited the work, NR also corrected a part of the article and FSh did statistical work.

\section{Funding}

This study was patronaged by a grant from Tran University of Medical Sciences. Grant number(46718_212_3_98)

\section{Availability of data and materials}

Participants of this study did not agree for their data to be shared publicly, so supporting data is not available.

\section{Ethics approval and consent to participate}

The present study was confirmed by Medical Research Ethics Committee of TUMS(IR.TUMS.MEDICINE.REC.1399.165).

\section{Consent for publication}

Not applicable.

\section{Competing interests}

The authors declare that they have no competing interests

Author Details

${ }^{1}$ Department of Community Nutrition, School of Nutritional Sciences and Dietetics, Tehran University of Medical Sciences (TUMS), Tehran, Iran. ${ }^{2}$ Department of Epidemiology and Biostatistics, School of Public Health, Tehran University of Medical Sciences (TUMS), Tehran, Iran. ${ }^{3}$ Department of Clinical Nutrition, School of Nutritional Sciences and Dietetics, Tehran University of Medical Sciences.

\section{References}

1. Mohammed MS, Sendra S, Lloret J, Bosch Systems and WBANs for Controlling Obesity. J Healthc Eng. 2018;2018:1564748.

2. Jafari-Adli Sh, Jouyandeh Z, Qorbani M, Soroush A, Larijani B, Hasani-Ranjbar S. Prevalence of obesity and overweight in adults and children in Iran; a systematic review. J Diabetes Metab Disord 2014;13(1):121. doi: 10.1186/s40200-014-0121-2.

3. Rashidy-Pour A, Malek M, Eskandarian R, Ghorbani R. Obesity in the Iranian population. Obes Rev.2009;10(1):2-6.

4. Hill J O, Wyatt H R, Peters J C. Energy Balance and Obesity. Circulation. 2012;126(1):126-32.

5. Hert M D, correll C.U, BoBes J, cetkovicH-BakMas M, coHen D, et al. Physical illness in patients with severe mental disorders.I. Prevalence, impact of medications and disparities in health care. World Psychiatry. 2011;10(1):52-77. 
6. Park E. Gender as a moderator in the association of body weight to smoking and mental health. Am J Public Health. 2009;99(1):146- 51.

7. Kaveh O, Peyrovi H. Exploring the Experiences of Iranian Women Regarding Obesity Self-Management: A Qualitative Study. J Med Sci.2019;7(14):2377-2383.

8. Kyrou, I., Randeva, H. S., Tsigos, C., Kaltsas, G., \& Weickert, M. O. (2018). Clinical problems caused by obesity. In Endotext [Internet]: MDText. com, Inc.

9. De Hert M, Correll CU, Bobes J, et al. Physical illness in patients with severe mental disorders. I. Prevalence, impact of medications and disparities in health care. World Psychiatry. 2011;10(1):52-77.

10. Simon GE, Von Korff M, Saunders K et al. Association between obesity and psychiatric disorders in the US adult population. Archives of General Psychiatry 2006; 63(7): 824-830.

11. Muhlhans B, Horbach T \& de Zwaan M. Psychiatric disorders in bariatric surgery candidates: a review of the literature and results of a German prebariatric surgery sample. General Hospital Psychiatry 2009; 31(5): 414421.

12. Westhuizen C, Wyatt G, Williams JK, Stein DJ, Sorsdahl K. Prevalence and predictors of mental disorders in intentionally and unintentionally injured emergency center patients. J Nerv Ment Dis. 2014;202(9):638-46.

13. Noorbala AA, Bagheri Yazdi SA, Yasamy MT, Mohammad K. Mental health survey of the adult population in Iran. Br J Psychiatry. 2004;184:70-3.

14. M C Zabala-Baños, A Segura, C Maestre-Miquel, M Martínez-Lorca, B Rodríguez-Martín, et al. Mental disorder prevalence and associated risk factors in three prisons of Spain. Rev Esp Sanid Penit. 2016;18(1):13-23.

15. Owen L, Corfe The role of diet and nutrition on mental health and wellbeing. Proc Nutr Soc. 2017;76(4):425426.

16. Nanri A, Eguchi M, Kuwahara K, Kochi T, Kurotani K, Ito R, et al. Macronutrient intake and depressive symptoms among Japanese male workers: the Furukawa Nutrition and Health Study. Psychiatry Res. 2014; 220(1-2):263-8.

17. Kuczmarski MF, Sees AC, Hotchkiss L, Cotugna N, Evans MK, Zonderman AB. Higher healthy eating Index2005 scores associated with reduced symptoms of depression in an urban population: findings from the healthy aging in neighborhoods of diversity across the life span (HANDLS) study. J Am Diet Assoc. 2010;110:383-9.

18. Jakulj, F.; Zernicke, K.; Bacon, S.L.; van Wielingen, L.E.; Key, B.L.; West, S.G.; Campbell, T.S. A high-fat meal increases cardiovascular reactivity to psychological stress in healthy young adults. J. Nutr. 2007, 137, 935939. [CrossRef] [PubMed].

19. Khankan J, Jazayeri Sh, Shidfar F, Sajadi Hezaveh Z, Hosseini AF, et al. The Relationship between NutrientRich Foods (NRF) Index as A Measure of Diet Quality and Malnutrition in Children. JNFS. 2019; 4(1):34-42.

20. Alkerwi Aa 2014. Diet quality concept. Nutrition. 30 (6): 613-618.

21. Fulgoni VL, Keast DR \& Drewnowski A 2009. Development and validation of the nutrient-rich foods index: a tool to measure nutritional quality of foods. Journal of nutrition. 139 (8): 1549- 1554.

22. Drewnowski A \& Fulgoni VL 2014. Nutrient density: principles and evaluation tools. American journal of clinical nutrition. 99 (5): 1223S-1228S.

23. MT Streppel, D Sluik, JF van Yperen, A Geelen, A Hofman, et al. Nutrient-rich foods, cardiovascular diseases and all-causemortality: the Rotterdam study. European journal of clinical nutrition. 2014;68;741-747. 
24. Streppel M T, Grootand L C P G M, Feskens E J M. Nutrient-rich foods in relation to various measures of anthropometry. Fam Pract. 2012;29(1):i36-43.

25. Mirmiran P, Hosseini Esfahani F, Mehrabi Y, Hedayati M, Azizi Reliability and relative validity of an FFQ for nutrients in theTehran Lipid and Glucose Study. Public Health Nutr. 2010;13(5):654-62.

26. Farhadnejad, H., Asghari, G., Mirmiran, P. \& Azizi, F. Dietary approach to stop hypertension diet and cardiovascular risk factors among 10- to 18-year-old individuals. Pediatric obesity13, 185-194, https://doi.org/10.1111/ijpo.12268 (2018).

27. Drewnowski A, Fulgoni V III. Nutrient profiling of foods: creating a nutrient-rich food index. Nutr Rev 2008;66:23-39.

28. Fulgoni VL III, Keast DR, Drewnowski A. Development and validation of the Nutrient Rich Foods Index: a tool to measure nutrient density of foods. J Nutr 2009;139:1549-54.

29. Fulgoni VL, Keast DR \& Drewnowski A 2009. Development and validation of the nutrient-rich foods index: a tool to measure nutritional quality of foods. Journal of nutrition. 139 (8): 1549- 1554.

30. Drewnowski A. The Nutrient Rich Foods Index helps to identify healthy, affordable foods. Am J Clin Nutr. 2010; 91(4):1095S-1101.

31. Arambepola C, Scarborough P, Rayner M. Validating a nutrient profile model. Public Health Nutr 2008;11:371-8.

32. Maillot M, Ferguson EL, Drewnowski A, Darmon N. Nutrient profiling can help identify foods of good nutritional quality for their price: a validation study with linear programming. J Nutr 2008;138:1107-13.

33. Drewnowski A 2009. Defining nutrient density: development and validation of the nutrient rich foods index. Journal of the American college of nutrition. 28 (4): 421S-426S.

34. US Food and Drug Administration. Code of Federal Regulations, Title 21, Food and drugs (vol 2). Chapter IFood and Drug Administration, Department of Health and Human Services. Part 101-Food Labeling- Table of Contents, Subpart A: General Provisions, Sec. 101.14. Health claims: General requirements and Subpart E -specific requirements for Health Claims. US Government Printing Office, revised as of 1 April 2002.

35. US Food and Drug Administration. Code of Federal Regulations. Sec 101.9, Nutrition labeling of food. 2007. Available from: http://www. accessdata. fda. goc/scripts/cdrh/cfdocs/cfcfr/cfrsearch. cfm?fr=109.9 (cited 8 February 2010).

36. Henry JD, Crawford JR (2005) The short-form version of the Depression Anxiety Stress Scales (DASS-21): Construct validity and normative data in a large non-clinical sample. Br J Clin Psychol44, 227-239.

37. Moghaddam MH, Aghdam FB, Jafarabadi MA, Allahverdipour H, Nikookheslat SD, Safarpour S. The Iranian version of international physical activity questionnaire (IPAQ) in Iran: Content and construct validity, factor structure, internal consistency and stability. World Appl Sci J 2012;18:1073-80.

38. Schweren L J S, Larsson H, Vinke P C, Li L, Kvalvik L G, et al. Diet Quality, Stress and Common Mental Health Problems: a Cohort Study of 121,008 Adults. Clin Nutr. 2020;30: 0261-5614(20)30327-7.

39. Naoko Takashima, Akihiko Katayama, Kazumi Dokai Mochimasu, Shuhei Hishii, Hiromi Suzuk, et al. A Pilot Study of the Relationship between Diet and Mental Health in Community Dwelling Japanese Women. Medicina, 2019;55(9):513.

40. Poorrezaeiana M, Siassi F, Qorbani M, Karimi J, Koohdani F, et al. Association of dietary diversity score with anxiety in women. Psychiatry Res. 2015;230(2):622-7. 
41. Poorrezaeian M, Siassi F, Milajerdi A, Qorbani M, Karimi J, ET AL. Depression is related to dietary diversity score in women: a cross-sectional study from a developing country. Ann Gen Psychiatry. 2017;16:39.

42. Meng L, Wang Y, Li T, Loo-Bouwman C A, Zhang Y, et al. Dietary Diversity and Food Variety in Chinese Children Aged 3-17 Years: Are They Negatively Associated with Dietary Micronutrient Inadequacy? Nutrients. 2018;10(11):1674.

43. S. Opie, C. Itsiopoulos, N. Parletta, A. Sanchez-Villegas, T.N. Akbaraly, et al. Dietary recommendations for the prevention of depression. Nutr Neurosci. 2017;20(3):161-171.

44. Gibson-Smith D, Bot M, Brouwer I.A, Visser M, Penninx B W.J.H. Diet quality in persons with and without depressive and anxiety disorders. J Psychiatr Res. 2018;106:1-7.

45. Natalie Parletta, Dorota Zarnowiecki, Jihyun Cho, Amy Wilson, Svetlana Bogomolova, et al. A Mediterraneanstyle dietary intervention supplemented with fish oil improves diet quality and mental health in people with depression: A randomized controlled trial (HELFIMED). Nutr Neurosci. 2019;22(7):474-487.

46. Benton, D., \& Donohoe, R. T. (1999). The effects of nutrients on mood. Public Health Nutrition, 2, 403-409.

47. Lang UE, Beglinger C, Schweinfurth N, Walter M, Borgwardt S. Nutritional aspects of depression. Cell Physiol Biochem. 2015;37:1029-43.

48. Schefft C, Kilarski LL, Bschor T, Köhler S. Efficacy of adding nutritional supplements in unipolar depression: a systematic review and meta-analysis. Eur Neuropsychopharmacol. 2017;27:1090-109.

49. Singh A, Trumpff C, Genkinger J, Davis A, Spann M, et al. Micronutrient Dietary Intake in Latina PregnantAdolescents and Its Association with Level ofDepression, Stress, and Social Support. Nutrients. 2017;9(11):1212.

50. Akhondzadeh S, Gerbarg P.L, Brown R.P. Nutrients for Prevention and Treatment of Mental Health Disorders. Psychiatr Clin North Am. 2013;36(1):25-36.

51. Knapp S, Mandell AJ, Bullard WP. Calcium activation of brain tryptophan hydroxylase. Life Sci. 1975;16:1583-93.

52. Grosso G, Galvano F, Marventano S, Malaguarnera M, Bucolo C, Drago F, Caraci F. Omega-3 fatty acids and depression: scientific evidence and biological mechanisms. Oxidative Med Cell Longev. 2014;2014:313570.

53. Sanchez-Villegas A, Henriquez P, Bes RM, Doreste J. Mediterranean diet and depression. Public Health Nutr. 2006;9:1104-9.

54. Bjelland, I.; Tell, G.S.; Vollset, S.E.; Refsum, H.; Ueland, P.M. Folate, vitamin B12, homocysteine, and the MTHFR 677C->T polymorphism in anxiety and depression: The Hordaland Homocysteine Study. Arch. Gen. Psychiatry 2003, 60, 618-626. [CrossRef] [PubMed]

55. Mattson, M.P.; Shea, T.B. Folate and homocysteine metabolism in neural plasticity and neurodegenerative disorders. Trends Neurosci. 2003, 26, 137-146. [CrossRef]

56. Tucker, K.L. Stress and nutrition in relation to excess development of chronic disease in Puerto Rican adults living in the Northeastern USA. J. Med. Investig. 2005, 52, 252-258. [CrossRef]

57. Harrison, F.E.; May, J.M. Vitamin C function in the brain: Vital role of the ascorbate transporter SVCT2. Free Radic. Biol. Med. 2009, 46, 719-730. [CrossRef] [PubMed]

58. Gareri, P.; Mattace, R.; Nava, F.; De Sarro, G. Role of calcium in brain aging. Gen. Pharmacol. 1995, 26, 16511657. [CrossRef]. 
59. Black, M.M. Zinc deficiency and child development. Am. J. Clin. Nutr. 1998, 68 (Suppl. 2), 464s-469s. [CrossRef] [PubMed].

60. Soltani H, Keim N.L, Laugero K.D. Diet Quality for Sodium and Vegetables Mediate Effects of Whole Food Diets on 8-Week Changes in Stress Load. Nutrients. 2018;10(11):1606.

61. Gonzalez M. J, Miranda-Massari R.J. Diet and Stress. Psychiatr Clin North Am. 2014;37(4):579-89.

62. de Baaij JH, Hoenderop JG, Bindels RJ. Magnesium in man: implications for health and disease. Physiol Rev 2015;95(1):1-46; [Research Support, Non-U S Government Review].

63. Seelig MS. Consequences of magnesium deficiency on the enhancement of stress reactions; preventive and therapeutic implications (a review). J Am Coll Nutr. 1994; 13: 429-446. PMID: 7836621.

64. Whyte KF, Addis GJ, Whitesmith R, Reid JL. Adrenergic control of plasma magnesium in man. Clinical Science. 1987; 72: 135-138. PMID: 3542342.

65. Cuciureanu MD, Vink R. Chapter 19: Magnesium and Stress. In: Vink R, ed. Magnesium in the Central Nervous System. University of Adelaide Press; 2015.

66. Murck H. Magnesium and affective disorders. Nutr Neurosci. 2002; 5: 375-389. https://doi.org/10.1080/ 1028415021000039194 PMID: 12509067.

67. Laugero KD, Falcon LM, Tucker KL. Relationship between perceived stress and dietary and activity patterns in older adults participating in the Boston Puerto Rican Health Study. Appetite. 2011;56:194-204.

68. Shabbir F, Patel A, Mattison C, Bose S, Krishnamohan R, Sweeney E, Sandhu S, Nel W, Rais A, Sandhu R, et al. Effect of diet on serotonergic neurotransmission in depression. Neurochem Int. 2013;62:324-9.

69. Crichton Honours G E(Hons), Murphy K J, Bryan J. Dairy intake and cognitive health in middle-aged South Australians. Asia Pac J Clin Nutr. 2010;19(2):161-71.

70. Scarmeas N, Stern Y, Tang MX, Mayeux R, Luchsinger JA. Mediterranean diet and risk for Alzheimer's disease. Ann Neurol. 2006;59:912-21.

71. Peltner J, Thiele S. Association between the Healthy Eating Index-2010 and nutrient and energy densities of German households' food purchases. Eur J Public Health. 2017 Jun 1;27(3):547-552.

72. Lutz, L.J.; Gaffney-Stomberg, E.; Williams, K.W.; McGraw, S.M.; Niro, P.J.; Karl, J.P.; Cable, S.J.; Cropper, T.L.; McClung, J.P. Adherence to the Dietary Guidelines for Americans Is Associated with Psychological Resilience in Young Adults: A Cross-Sectional Study. J. Acad. Nutr. Dietet. 2017, 117, 396-403. [CrossRef] [PubMed]

73. Kennedy DO, Haskell CF. Vitamins and cognition what is the evidence? Drugs 2011;71(15):1857-971.

74. Rucklidge JJ, Kaplan BJ, Mulder RT. What if nutrients could treat mental illness? Aust N Z J Psychiatry 2015;49(5):407-8. 